\title{
Preliminary Report on the Competitive Phagocytosis between Bone Marrow and Alveolar Macrophages
}

\author{
Elena Ghezzi* and Kathryn Michels, $\mathrm{PhD} \dagger$ \\ University of Michigan Medicine - Microbiology and Immunology Department \\ *To whom correspondence should be addressed: etghezzi@umich.edu \\ $\dagger$ †kathrmic@umich.edu
}

\begin{abstract}
Asthma is a widespread and debilitating disease characterized by lung inflammation and triggered by environmental allergen exposure. The severity of the reaction to these antigens is determined by the immune response of different white blood cells (WBC) present in the lung: alveolar macrophages (AM) and recruited bone marrow macrophages (BMM). Previous studies suggest that AM often play an anti-inflammatory role and protect against asthma while BMM have proinflammatory tendencies. The hypothesis of this project is that when both AM and BMM are present in the lung and come into contact with allergens, the BMM will engulf more of these particles due to their hyperreactive immune response. To test this hypothesis, BMM were extracted from the tibia and femur of a mouse and then plated with beads, in order to mimic the lung conditions during allergen exposure. AM were then plated alongside the BMM for comparison of particle engulfment. The phagocytosis of the macrophages was measured in order to determine the percentage of each WBC type that engulfed beads in differing conditions. The data from this preliminary study reveal that the BMM phagocytose more beads when AM are present, suggesting a synergistic effect on hyperreactive immune response. Future studies would further define the relationship between competing WBC types during asthma.
\end{abstract}

\section{Introduction}

Asthma is a crippling chronic disease with a large global health burden and has increased in prevalence in the $21^{\text {st }}$ century. ${ }^{1}$ Typical asthma symptoms can vary in severity and include wheeze, cough, and difficulty breathing. ${ }^{2}$ This disease progresses upon exposure to a respiratory irritant such as an environmental allergen which is recognized by cells that line the airway of the lungs, known as the airway epithelium. ${ }^{1}$ Airway epithelial cells, along with other white blood cells, secrete small immune-activating proteins, known as cytokines. Inflammatory cytokines activate dendritic cells which in turn activate $\mathrm{T}$ cells, resulting in an immune response that causes the symptoms of asthma. ${ }^{3}$

Lung airways are regularly exposed to substances that can threaten the respiratory system. Alveolar macrophages (AM) are a type of white blood cell found within alveoli (the air sacs of the lung) that must evaluate entities entering the airway and maintain immune equilibrium within the lungs. AM have different effects on the immune system depending on origin. AM that are recruited from the bloodstream tend to be proinflammatory, while AM that live full time in lung tissue show anti-inflammatory tendencies by reducing $\mathrm{T}$ cell activation. ${ }^{4}$ Reduced $\mathrm{T}$ cell activation lowers the hyperactive immune response and limits asthma symptoms. It has been shown that asthma development impairs AM function, decreasing AM ability to capture particles that they encounter in the airway, possibly impacting how the other cells of the immune system will respond and causing immune imbalance. ${ }^{5}$

Recent studies have observed and compared the inflammatory role of AM in comparison to another type of WBC or monocytes, Bone Marrow Macrophages (BMM) which are recruited into the lung once an irritant is present. ${ }^{4}$ During 
inflammation, locally-derived AM were observed to play an anti-inflammatory role by inhibiting the recruitment of other white blood cells to the lung, whereas BMM were shown to promote inflammation. ${ }^{5}$ The interaction of these two WBC forms in the lung may affect the manifestation of asthma symptoms, as the cells sometimes exhibit opposing effects upon lung exposure to irritants. Recent studies have observed that when the lung's exposure to allergens is minimal, the BMM play a minor role as they are only heavily recruited once the antigen threatens to lead to infection in lung tissue. When the threat of infection is at a minimum, the AM dominate the lung's immune response and engulf the allergens while suppressing the genes that signal for additional proinflammatory cells. ${ }^{6}$ Once acute inflammation occurs in the lungs, a signal from the epithelial cells allows for the migration of BMM to the lungs and the AM undergo a change in phenotype and begin to alter their immune response, transforming from naïve AM into a more inflammatory state. This new population of AM engulf fewer antigens and increase their expression of cytokines. ${ }^{7}$ This modification in phenotype of the naïve AM is associated with reduced anti-inflammatory properties and protection against asthma symptoms. $^{7}$

Because BMM tend to play a hyperreactive immunological role while AM suppress inflammation in the context of allergy-mediated lung inflammation, we hypothesize that BMM are able to phagocytose more beads, which represent irritants in the lung, as compared to AM. The phagocytosis of these small fluorescent beads is representative of the action that these cell types take in response to the presence of allergens in the lungs as AM and BMM engulf antigens and then proceed to either promote or suppress the following immune response. The greater BMM phagocytosis of beads would lead to their increased immune response and prevention of the AM role to maintain immune equilibrium within the airways, similar to symptoms following exposure to irritants.

\section{Methods}

Bone marrow macrophages were harvested from male Balb/C mice 6 months of age bred in Jackson Laboratories (Bar Harbor, ME). Bone marrow was harvested by flushing RPMI (Lonza Walkersville, Walkersville MD, IUSA) through the tibia and femur. The bone marrow was resuspended in a filter-sterilized bone marrow macrophage media (BMM) that is composed of 52\% RPMI 1640, 31\% L-cell sup (generated on-site and kept in freezer), 16\% fetal bovine serum
(FBS) (Atlas Biologicals, Fort Collins, CO, USA) and 1\% Penn strep in a $5 \mathrm{~mL}$ aliquot (Mediatech, Inc. Manassas, VA, USA). The initial concentration of the bone marrow macrophages was determined by manual counting on a hemocytometer and then the solution was plated onto $100 \mathrm{~mm}$ cell culture dishes at a concentration between $5 \times 10^{5}$ and $1 \times 10^{6}$ cells $/ \mathrm{mL}$. The cells were incubated in a $37^{\circ} \mathrm{C} 5 \% \mathrm{CO}_{2}$ incubator and were fed on day 3 or 4 with $10 \mathrm{~mL}$ of BMM. Bone marrow macrophages were collected off the culture plates on day 7 by two successive incubations with $0.25 \%$ trypsin (Mediatech Inc., Manassas, VA, USA) and gentle scraping, followed by rescue with RPMI solution. Then the cells were isolated from the solution via centrifugation. The concentration of the remaining isolated macrophages was measured using the manual counting process on the hemocytometer so that multiple samples at a concentration of $2 \times 10^{4}$ cells/ $100 \mu \mathrm{L}$ could be dispensed onto a 96 flat well plate. In later trials, these samples were plated instead onto Teflon wells as $100 \mu \mathrm{L}$ at a concentration of $8 \times 10^{5}$ cells $/ \mathrm{mL}$ in order to avoid the loss of cells due to adherence. In order to observe the immune response of the bone marrow macrophages, they were plated with varying concentrations of Fluoresbrite ${ }^{\circledR}$ YG $1.0 \mu \mathrm{m}$ microspheres (Polysciences, Inc., Warrington, PA, USA). The beads, a model for the various antigens a macrophage may encounter, were distributed at concentrations of $0.5 \%$ or $0.1 \%$ for the first phagocytosis assays. After analysis of the results, it was determined that $0.1 \%$ was an ideal concentration since the $0.5 \%$ beads were too concentrated and interfering with the determination of the number of bead + cells as they bind extracellularly to macrophages. In later trials, $0.04 \%$ Trypan Blue solution (Sigma-Aldrich Corporation. St. Louis, MO, USA) was added to these mixtures of beads and BMM in order to quench the extracellular fluorescence that results when beads bind to the outside of the BMM and falsely mimic engulfment. The experiments in the wells containing beads and bone marrow macrophages were allowed to proceed for various segments of time, for 1 and 4 hours in some trials or 2 and 4 hours in others, prior to data analysis. Following the completion of the phagocytosis within each well, the individual events were then collected with the NovoCyte Flow Cytometer (Acea Biosciences, Inc. San Diego, CA, USA). The recorded data was then analyzed using the FloJo Software (FlowJo LLC, Ashland, Oregon, USA) in order to evaluate the relationship among certain variables (forward scatter, side scatter, and fluorescence of the cells) of both groups of macrophages, those that engulfed and those that fail to engulf the beads, present in the solutions. The FloJo analysis allowed for the determination of 
bead + cells present in each well. Once a solid methods protocol was established and certain variables were determined, alveolar macrophages were collected and plated in Teflon cells with beads in order to compare their phagocytosis activity to that of BMM. Samples $8 \times 10^{5}$ cells $/ \mathrm{mL}$ of AM were plated separately with $0.1 \%$ beads and then $4 \times 10^{5}$ cells $/ \mathrm{mL}$ at a volume of $50 \mu \mathrm{L}$ of AM were plated with the same concentration of BMM and $0.1 \%$ beads. These experiments were allowed to run for 2 hours and characterized with NovoCyte Flow Cytometry in a similar manner as the original BMM wells were measured. Once the data were collected it was analyzed and compared in tables on excel, using the data for cell frequency and percentage of bead + BMM or AM that was generated from FlowCytometry and FloJo. Student T-tests were run on the samples in order to determine the statistical significance of the difference in phagocytosis activity for AM compared to BMM.

\section{Discussion and Results}

In the initial experiment, bone marrow macrophages were plated with $0.5 \%$ concentration of fluorescent microsphere beads in both $37^{\circ} \mathrm{C}$ and $4^{\circ} \mathrm{C}$. The number of cells present in the wells of both groups and the percentage of those cells that engulfed the beads (Bead + BMM) were analyzed and compared. The Flow Cytometry measurements and FloJo analysis indicate that a higher count of BMM cells were present in the $4^{\circ} \mathrm{C}$ wells compared to the wells kept at $37^{\circ} \mathrm{C}$ (Figure 1).

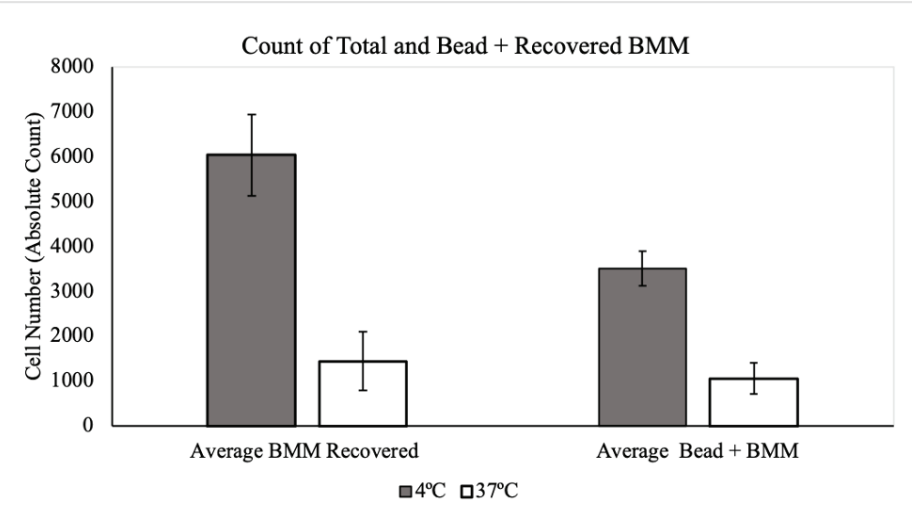

Figure 1. The mean count and standard error of the mean $(n=3)$ of BMM present in both of the well plate storage conditions. The frequency of macrophages that appeared to engulf beads presented as a count with a standard error of the mean ( $n=3$ for each group). These data are a representative result of two different experiments that led to the same conclusion regarding ideal experiment conditions.
This discrepancy in absolute cell number despite plating at identical concentrations suggests that in a warmer environment the BMM can undergo the biological processes that allow them to adhere to the bottom of the flat well plates. When cells adhere to the bottom of the wells, it is difficult to recover them and analyze them. We also found that cells incubated at $4^{\circ} \mathrm{C}$ had a high frequency of bead labeling. These results suggest that large numbers of beads become stuck to the outside of the cell because cells are not able to phagocytose beads in cold temperatures. As a result, in these conditions, it was difficult to tell the difference between cells that phagocytosed beads and those that had beads stuck to the outside. We decided to modify the procedure by; 1 . using Teflon wells, which will prevent cells from sticking to the bottom of the plate 2. reducing the bead concentration to $0.1 \%$ to limit background bead binding and 3. treating the cells with Trypan Blue, which will reduce the signal from beads attached to the outside of cells but will not affect the fluorescence of beads on the inside of cells. These experimental changes reduced nonspecific bead binding for both types of macrophages, resulting in improved accuracy in the measurement of cells that phagocytosed beads (Figures $2 \& 3$ ).

Both the AM and BMM control groups in the modified
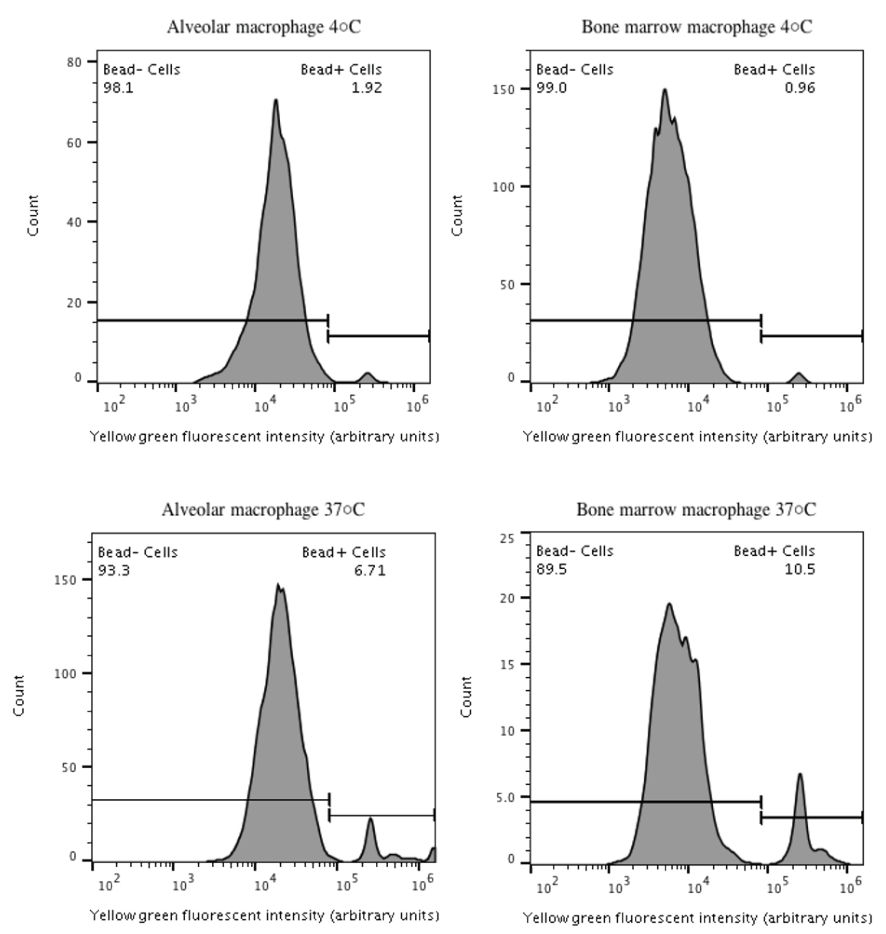

Figure 2. AM and BMM bead phagocytosis as determined by flow cytometry. Representative plot of data used to determine bead binding background $\left(4^{\circ} \mathrm{C}\right)$ and phagocytosis $\left(37^{\circ} \mathrm{C}\right)$. 


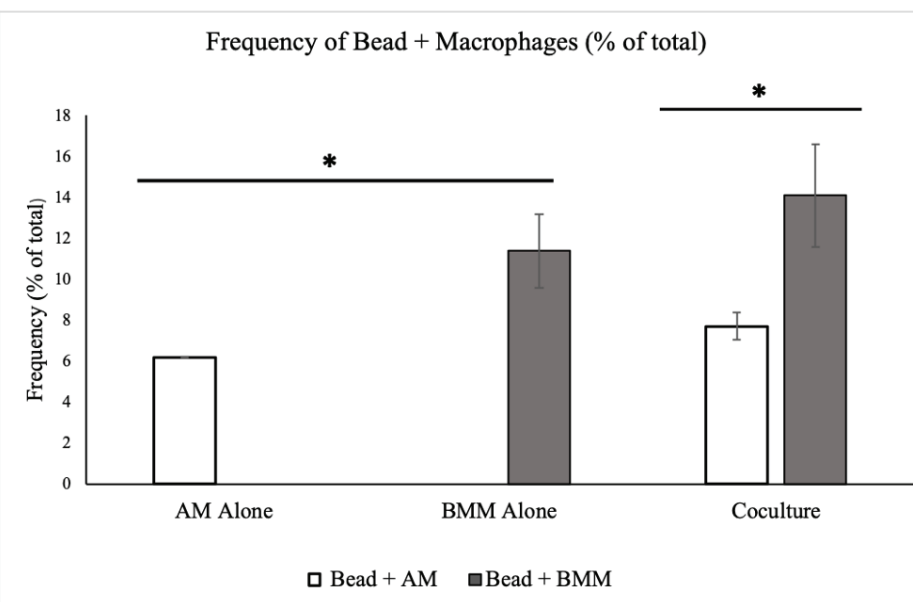

Figure 3. The mean relative percentages of phagocytosis and standard error of mean for each cell type. $\mathrm{N}=3$ for each group. Statistically significant differences in relative frequencies of Bead + AM and BMM were observed when plated together and when plated alone $(* \mathrm{P}<0.05)$. The error bars on AM alone are too small to be seen (+/- 0.03$)$. These data are representative of two separate experiments that led to the same conclusion regarding relative macrophage engulfment of beads.

experiments displayed a small and similar background percentage, indicating that these changes allowed for a more representative analysis of the occurrences within the wells.

Once it was observed that BMM engulf the beads at $37^{\circ} \mathrm{C}$ under the ideal determined conditions, their behavior was compared to that of AM as the two were plated with beads both separately and together. When analyzed separately both $\mathrm{AM}$ and $\mathrm{BMM}$ display phagocytosis of beads in $37^{\circ} \mathrm{C}$. However, the relative percentage of phagocytosis is not significantly different from their percentage of engulfment when present together. This indicates that their activity is not changed regardless of whether the other cell type is present to compete for engulfment. When plated together, the BMM display a higher percentage of engulfment of beads in comparison to the AM present in the same wells (Figure 3 ). The discrepancy between the relative frequency of bead + macrophages present in the same wells was determined to be a statistically significant difference through a student t-test analysis that correlated to a p-value $<0.05$ (Figure 3).

This significant contrast in reactivity to the presence of the beads may suggest a difference in the immune response of these two cell types in response to allergen exposure, a typical asthma trigger. It is possible that because the BMM tend to engulf more beads, the representations of allergens, once they are present in the lung due to inflammation, they tend to contribute to and dominate the proinflammatory response in comparison to the AM that phagocytose less. This greater contact between BMM and the increased concentration of allergens during a threat of infection allows for cytokine signaling of additional pro-inflammatory cells, possibly superseding any anti-inflammatory action of AMs. Continuation and advancement of this study is necessary to corroborate this theory.

\section{Further Directions}

The results of this preliminary study suggest the greater hyperreactive immune role of BMM in comparison to AM in the lung. In order to further validate the significant result that BMM engulf more allergens when plated with AM, the same bead model with AM and BMM could be replicated again in future studies. In future studies, we can conjugate allergens directly to microspheres, allowing us to study cellular responses in the context of allergen-mediated activation. This experiment compared naïve AM to BMM and could be advanced by plating BMM and allergen-exposed $\mathrm{AM}$ together with beads to measure their phagocytosis. Other studies suggest that while naïve AM tend to be anti-inflammatory, AM that are exposed to allergens may transform and display a pro-inflammatory response. While it is suggested that BMM are more hyperreactive than naïve $\mathrm{AM}$, this advancement could study whether this relationship changes with previous allergen exposure. Once this model has been further characterized in vitro, we can expand the study by injecting microspheres directly into the airways of mice and determine if differential phagocytosis also occurs in vivo. The study of the interplay of these $\mathrm{WBC}$ and their varying responses to the antigens could contribute to the immunological understanding of the pathogenesis of asthma.

\section{References}

1. C. Draijer, M. Peters-Golden, Alveolar Macrophages in Allergic Asthma: the Forgotten Cell Awakes. Curr. Allergy Asthma Rep. 17, 12 (2017), doi:10.1007/s11882017-0681-6.

2. H. S. Zahran, C. M. Bailey, S. A. Damon, P. L. Garbe, P. N. Breysse, Vital signs: Asthma in children - United States, 2001-2016. Morb. Mortal. Wkly. Rep. 67, 149155 (2018), doi:10.15585/mmwr.mm6705e1.

3. H. Hammad, B. N. Lambrecht, Dendritic cells and epithelial cells: Linking innate and adaptive immu- 
nity in asthma. Nat. Rev. Immunol. 8, 193-204 (2008), doi:10.1038/nri2275.

4. Z. Zasłona, S. Przybranowski, C. Wilke, N. van Rooijen, S. Teitz-Tennenbaum, J. J. Osterholzer, J. E. Wilkinson, B. B. Moore, M. Peters-Golden, Resident Alveolar Macrophages Suppress, whereas Recruited Monocytes Promote, Allergic Lung Inflammation in Murine Models of Asthma. J. Immunol. 193, 4245-4253 (2014), doi:10.4049/jimmunol.1400580.

5. A. M. Fitzpatrick, F. Holguin, W. G. Teague, L. A. S. Brown, Alveolar macrophage phagocytosis is impaired in children with poorly controlled asthma. J. Allergy Clin. Immunol. 121, 1372-1378 (2008), doi:10.1016/j. jaci.2008.03.008.

6. Y. G. Lee, J. J. Jeong, S. Nyenhuis, E. Berdyshev, S.
Chung, R. Ranjan, M. Karpurapu, J. Deng, F. Qian, E. A. B. Kelly, N. N. Jarjour, S. J. Ackerman, V. Natarajan, J. W. Christman, G. Y. Park, Recruited alveolar macrophages, in response to airway epithelial-derived monocyte chemoattractant protein 1/CCL2, regulate airway inflammation and remodeling in allergic asthma. Am. J. Respir. Cell Mol. Biol. 52, $772-784$ (2015), doi:10.1165/ rcmb.2014-0255OC.

7. T. Naessens, S. Vander Beken, P. Bogaert, N. Van Rooijen, S. Lienenklaus, S. Weiss, S. De Koker, J. Grooten, Innate imprinting of murine resident alveolar macrophages by allergic bronchial inflammation causes a switch from hypoinflammatory to hyperinflammatory reactivity. Am. J. Pathol. 181, 174-184 (2012), doi:10.1016/j.ajpath.2012.03.015. 